Bull. Austral. Math. Soc.

$52 \mathrm{C} 05,52 \mathrm{~A} 40,52 \mathrm{~A} 10$

VOL. 58 (1998) [159-166]

\title{
SOME INEQUALITIES FOR PLANAR CONVEX SETS CONTAINING ONE LATTICE POINT
}

\author{
M.A. Hernández Cifre and S. Segura Gomis
}

\begin{abstract}
We obtain two inequalities relating the diameter and the (minimal) width with the area of a planar convex set containing exactly one point of the integer lattice in its interior. They are best possible. We then use these results to obtain some related inequalities.
\end{abstract}

\section{INTRODUCTION AND RESULTS}

Let $K$ be a compact convex set in the Euclidean plane $E^{2}$, having area $A(K)=A$, (minimal) width $\omega(K)=\omega$ and diameter $d(K)=d$.

In [6], Scott established two "dual" inequalities, relating $d, \omega$ and $A$ for compact convex sets containing no points of the integer lattice $\mathbf{Z}^{2}$ in its interior:

$$
\begin{gathered}
(\omega-1) A \leqslant \frac{1}{2} \omega^{2} \\
(d-1) A \leqslant \frac{1}{2} d^{2}, \text { providing } d \leqslant 2 .
\end{gathered}
$$

In this paper we prove two similar inequalities, but in the case that $K$ contains one lattice-point of $\mathbf{Z}^{2}$ in its interior, and some related inequalities.

Let us define the family of the triangles $T_{\varepsilon}$ :

$$
\mathcal{T}=\left\{\operatorname{conv}\left\{(-2,-1),(1+\varepsilon,-1),\left(\frac{1-\varepsilon}{1+\varepsilon}, \frac{2}{1+\varepsilon}\right)\right\} / \varepsilon \in[0, \sqrt{2}-1]\right\} .
$$

Note that the triangles of the family $\mathcal{T}$ are the triangles "intermediate between" the isosceles triangles $T_{0}$ and $T_{\sqrt{2}-1}$, which are shown in Figure 1.

\footnotetext{
Received 19th February, 1998

The first author's work was supported by an FPPI Predoctoral Grant, Universidad de Murcia, 1996 and by Consejería de Cultura y Educación (C.A.R.M.) COM-05/96MAT. The second author's work was partially supported by a DGICYT Grant No. PB94-0750-C02-02 and by Consejería de Cultura y Educación (C.A.R.M.) COM-05/96MAT.
}

Copyright Clearance Centre, Inc. Serial-fee code: $0004-9729 / 98 \quad \$ A 2.00+0.00$. 


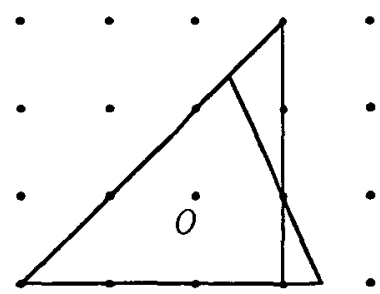

Figure 1: Extremal triangles

Now, we may establish the following theorems:

THEOREM 1 . If $K$ contains the origin $O$, but no other point of the integer lattice in its interior, then

$$
(\omega-\sqrt{2}) A \leqslant \frac{\sqrt{2}}{2} \omega^{2}
$$

with equality when and only when $K=T_{\varepsilon} \in \mathcal{T}$, for all $\varepsilon \in[0, \sqrt{2}-1]$ (up to congruence).

THEOREM 2. If $K$ contains the origin $O$, but no other point of $\mathbf{Z}^{2}$ in its interior, and $d \leqslant 2 \sqrt{2}$, then

$$
(d-\sqrt{2}) A \leqslant \frac{\sqrt{2}}{2} d^{2}
$$

with equality when and only when $K$ is a square of side length 2.

COROLLARY 1 . If $K$ contains the origin $O$, but no other point of $Z^{2}$ in its interior, then

$$
(\omega-\sqrt{2}) d^{2} \leqslant 2 \sqrt{2} A
$$

with equality when and only when $K=T_{\varepsilon} \in \mathcal{T}$, for all $\varepsilon \in[0, \sqrt{2}-1]$ (up to congruence).

Corollary 2. If $K$ contains the origin $O$, but no other point of $\mathbf{Z}^{2}$ in its interior, then

$$
(\omega-\sqrt{2}) A \leqslant \frac{3 \sqrt{2}}{2}+2
$$

with equality when and only when $K=T_{\sqrt{2}-1}$ (up to congruence).

\section{SOME PRELIMINARY RESULTS}

We shall require the following lemmas: 
LEMma 1. Let $\mathcal{Q}$ be the quadrilateral with vertices $X Y Z U$. Let $X Z=t$, and $\delta$ be the width of $\mathcal{Q}$ in a direction perpendicular to $X Z$. Then, any square which is inscribed in $\mathcal{Q}$ and has a vertex on each side of $\mathcal{Q}$ has side length $s$, satisfying:

$$
s \geqslant \frac{t \delta}{t+\delta}
$$

Proof: Let $A, B, C, D$ be the vertices of the inscribed square, which lie on the sides $U X, X Y, Y Z, Z U$ respectively (see Figure 2 ).

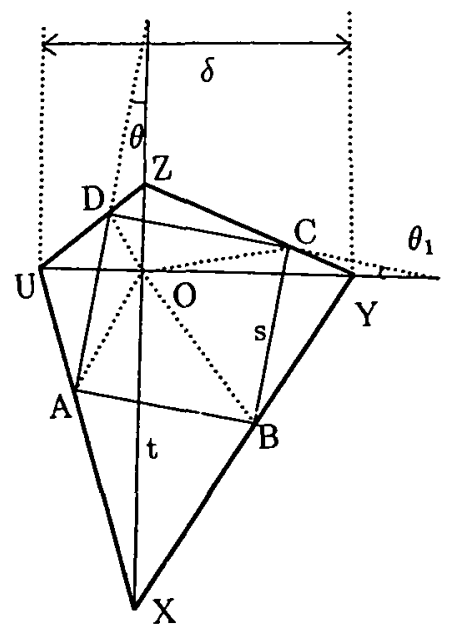

Figure 2

Let $A D$ make an angle $\theta$ with $X Z$, let $D C$ make an angle $\theta_{1}$ with $U Y$, and let $X Z$ meet $U Y$ in $O$.

Now, it is clear that the area of $\mathcal{Q}$ is $t \delta / 2$. But this area is also given by adding the areas of quadrilaterals $O D Z C, O B X A$ to the areas of quadrilaterals $O C Y B, O A U D$.

Then, we obtain:

$$
\frac{1}{2} t \delta=\frac{1}{2} s t \cos \theta+\frac{1}{2} s \overline{U Y} \cos \theta_{1} \leqslant \frac{1}{2} s(t \cos \theta+\delta) .
$$

So,

$$
s \geqslant \frac{t \delta}{t \cos \theta+\delta} \geqslant \frac{t \delta}{t+\delta}
$$

Lemma 2. Let $A, B, C, D$ be the vertices of a square with side length $s$, and let $\mathcal{Q}$ be the quadrilateral $X Y Z U$, with sides $X Y, Y Z, Z U, U X$, passing through $B, C, D$ $A$ respectively. Let $d_{1} \leqslant d_{2}$ be its diagonals. Then, $d_{1} \leqslant 2 s$. 
PRoOF: Using Steiner symmetrisation (see [3], Lemma 3), it is easy to see that $\mathcal{Q}$ can be transformed into a "kite" (a quadrilateral which is symmetric with respect to one of its diagonals) $\mathcal{Q}^{\prime}$, such that

(i) $\mathcal{Q}^{\prime}$ has its symmetry axis along the line $x=s / 2$ (for a suitable choice of coordinate axis).

(ii) The sides of $\mathcal{Q}^{\prime}$ pass through $A, B, C, D$ respectively.

(iii) The minor diagonal of $\mathcal{Q}^{\prime}, d_{1}^{\prime}$, satisfies $d_{1}^{\prime} \geqslant d_{1}$.

It is clear that for $\mathcal{Q}^{\prime}, d_{1}^{\prime} \leqslant 2 s$, and hence, $d_{1} \leqslant 2 s$.

\section{PRoOF OF THEOREM 1}

Let $r=r(K)$ be the inradius of $K$. It is shown in [5] that for any bounded convex set $K$,

$$
(\omega-2 r) A \leqslant \frac{\sqrt{3}}{3} \omega^{2} r
$$

So, it follows that if $r \leqslant \sqrt{2} / 2$, then

$$
(\omega-\sqrt{2}) A \leqslant(\omega-2 r) A \leqslant \frac{\sqrt{3}}{3} \omega^{2} r \leqslant \frac{\sqrt{3}}{3} \frac{\sqrt{2}}{2} \omega^{2}<\frac{\sqrt{2}}{2} \omega^{2} .
$$

Hence, we may assume that $K$ contains a disc $D_{\varepsilon}$ of radius $\frac{\sqrt{2}}{2}+\varepsilon, \varepsilon>0$.

It is no loss of generality to assume that the centre of $D_{\varepsilon}$ is interior to the square with vertices $Q_{1}=(1,1), Q_{2}=(-1,1), Q_{3}=(-1,-1), Q_{4}=(1,-1)$ (we suppose that $\left.\operatorname{int}(K) \cap \mathbf{Z}^{2}=\{O\}\right)$.

Let $\mathcal{K}_{\varepsilon}$ denote the class of compact convex sets $K$ containing a disc $D_{\varepsilon}$ placed as above, with $\operatorname{int}(K) \cap \mathbf{Z}^{2}=\{O\}$. It will be sufficient to prove the theorem for $\mathcal{K}_{\varepsilon}$.

Let now $\varepsilon>0$ be given. Since $Q_{i}, i=1,2,3,4$ are not interior to the sets $\mathcal{K}_{\varepsilon}$, we deduce that $\mathcal{K}_{\varepsilon}$ is uniformly bounded in the plane. As $\sqrt{2}(\omega-\sqrt{2}) A / \omega^{2}$ is a continuous function of $\omega, A$ for $\omega>0$, Blaschke's selection theorem [1] guarantees the existence of a maximal set in $\mathcal{K}_{\varepsilon}$, that is, a set $K \in \mathcal{K}_{\varepsilon}$ for which $\sqrt{2}(\omega-\sqrt{2}) A / \omega^{2}$ is as large as possible.

Let $f(\omega)=\sqrt{2} \omega^{2} / 2(\omega-\sqrt{2})$. As $f(\omega)$ is a decreasing function of $\omega$ (since $\omega \leqslant$ $1+\sqrt{2}<2 \sqrt{2}$, see [7]), we seek to obtain $K$ by initially making $\omega$ and $A$ as large as possible.

Let $N, S, E, W$ denote the lattice points $(0, \pm 1),( \pm 1,0)$ respectively. As $N, S, E, W \notin \operatorname{int}(K)$, and $K$ is convex, $K$ is bounded by lines through these points. These lines can form a convex quadrilateral $\mathcal{Q} \supseteq K$ or determine a triangular region $T \supseteq K$. In any case, $A(\mathcal{Q}) \geqslant A(K)$ and $A(T) \geqslant A(K)$. 


\section{The Triangle Case:}

Since in any triangle the width $\omega$ is in a direction perpendicular to the longest side, $A(T)=d \omega / 2$.

In [8], it is proved that if $K$ contains no point of the rectangular lattice with basis $\{(u, 0),(0, v)\}$, then

$$
(\omega-u)(d-v) \leqslant u v .
$$

Let us consider the lattice $\Gamma$ formed by the points

$$
\Gamma=\left\{(m, n) \in \mathbf{Z}^{2} / m+n \text { is an odd number }\right\},
$$

which we can identify with the lattice $\sqrt{2} \mathbf{Z}^{2}$ with basis $\{(\sqrt{2}, 0),(0, \sqrt{2})\}$. Then, $\operatorname{int}(K) \cap \sqrt{2} Z^{2}=\emptyset$, and by $(1),(\omega-\sqrt{2})(d-\sqrt{2}) \leqslant 2$. But this is easily seen to be equivalent to

$$
d \leqslant \sqrt{2} \frac{\omega}{\omega-\sqrt{2}} .
$$

Since the triangle $T$ contains no points of $\sqrt{2} \mathbf{Z}^{2}$, then the inequality (2) holds for $T$. Then

$$
A(T)=\frac{1}{2} d(T) \omega(T) \leqslant \frac{\sqrt{2}}{2} \frac{\omega(T)^{2}}{\omega(T)-\sqrt{2}} .
$$

But as $\operatorname{int}(T) \cap \sqrt{2} \mathbf{Z}^{2}=\emptyset$, we have [3]

$$
\omega(T) \leqslant \sqrt{2} \frac{1}{2}(2+\sqrt{3})<2 \sqrt{2}
$$

and hence,

$$
A \leqslant A(T) \leqslant \frac{\sqrt{2}}{2} \frac{\omega(T)^{2}}{\omega(T)-\sqrt{2}} \leqslant \frac{\sqrt{2}}{2} \frac{\omega^{2}}{\omega-\sqrt{2}} .
$$

And equality holds when and only when the equality holds in (2) and $K$ contains one lattice point in its interior, that is, when and only when $K$ is a triangle of diameter $d$ and width $\omega=\sqrt{2} d /(d-\sqrt{2})$, containing a unique lattice point in its interior. Hence, equality holds when and only when $K=T_{\varepsilon} \in \mathcal{T}$ (up to congruence).

\section{The Quadrilateral Case:}

Let quadrilateral $\mathcal{Q} \equiv X Y Z U$ have just one of the lattice points $N, S, E, W$ on each side. Let $X Z=t$ be its major diagonal, and let the width of $\mathcal{Q}$ in a direction perpendicular to $X Z$ be $\delta$.

Then, Lemma 1 assures us that $\sqrt{2} \geqslant t \delta /(t+\delta)$, which is equivalent to the inequality

$$
t \leqslant \sqrt{2} \frac{\delta}{\delta-\sqrt{2}}
$$


Further, the area of $\mathcal{Q}$ is given by

$$
A(\mathcal{Q})=\frac{1}{2} t \delta \leqslant \frac{\sqrt{2}}{2} \frac{\delta^{2}}{\delta-\sqrt{2}} .
$$

Note that $\delta \leqslant U Y$ (minor diagonal), and applying Lemma $2, U Y \leqslant 2 \sqrt{2}$. Hence, $\delta \leqslant 2 \sqrt{2}$.

Since $\omega<\omega(\mathcal{Q})<\delta \leqslant 2 \sqrt{2}$ and $\delta^{2} /(\delta-\sqrt{2})$ is a decreasing function of $\delta$, we have finally:

$$
A \leqslant A(\mathcal{Q})<\frac{\sqrt{2}}{2} \frac{\omega(\mathcal{Q})^{2}}{\omega(\mathcal{Q})-\sqrt{2}} \leqslant \frac{\sqrt{2}}{2} \frac{\omega^{2}}{\omega-\sqrt{2}} .
$$

This inequality is strict for a non-degenerate quadrilateral. This completes the proof of Theorem 1.

\section{Proof of Theorem 2}

Let $f(d)=\sqrt{2} d^{2} / 2(d-\sqrt{2})$. Again, $f(d)$ is a decreasing function of $d$ for $d \leqslant 2 \sqrt{2}$. A set $K$ for which $\sqrt{2}(d-\sqrt{2}) / d^{2}$ is as large as possible will be called a maximal set. The existence of such a maximal set is guaranteed by Blaschke's selection theorem [1], as the sets of diameter $d \leqslant 2 \sqrt{2}$ can be placed in a bounded portion of the plane.

Let us consider again the lattice $\Gamma$ defined in the proof of Theorem 1 . Then, $K$ contains no lattice-points of $\Gamma \equiv \sqrt{2} Z^{2}$ in its interior. It is shown in [4] that $A \leqslant \sqrt{2} \lambda d$ $(\lambda \approx 1.144)$, with equality when and only when $K=K^{*}$ is the intersection set of the disc $x^{2}+y^{2} \leqslant d^{2} / 4$ and the square with side $\sqrt{2} \sqrt{2}=2$.

Using the notation of the Figure 3 , and noting that $d=2 \sec \theta$, we have:

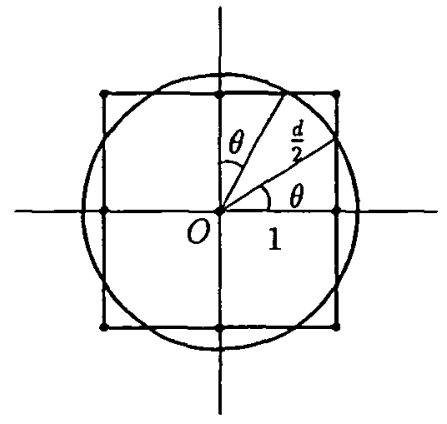

Figure 3

$$
A\left(K^{*}\right)=2 \sec ^{2} \theta\left(\frac{\pi}{2}-2 \theta+\sin 2 \theta\right), \text { where } 0 \leqslant \theta \leqslant \frac{\pi}{4} .
$$

Since $f(d)$ is a decreasing function of $d$ for $d \leqslant 2$, it will be sufficient to show that $A\left(K^{*}\right) \leqslant f(2 \sqrt{2})=4$, because then, $A \leqslant A\left(K^{*}\right) \leqslant f(2 \sqrt{2}) \leqslant f(d)$. 
But $A\left(K^{*}\right) \leqslant 4$ is equivalent to

$$
g(\theta)=\frac{\pi}{2}-2 \theta+\sin 2 \theta-2 \cos ^{2} \theta \leqslant 0 .
$$

Since $g^{\prime}(\theta)>0$ for the given range of $\theta$, we have $g(\theta) \leqslant g(\pi / 4)=0$.

It follows that $A \leqslant \sqrt{2} d^{2} / 2(d-\sqrt{2})$, with equality when and only when $d=2 \sqrt{2}$, and this occurs when $K=K^{*}$ is a square of side 2 .

It is easy to see that Theorem 2 fails for $d>2 \sqrt{2}$. We can take, for example, the intersection set of the isosceles triangle $T_{0}$ (shown in Figure 1), and the disc $x^{2}+y^{2} \leqslant r^{2}$, for any $r \in(\alpha, \sqrt{5})(\alpha \approx 1.4813)$.

\section{Proof of the Corollaries}

Corollary 2 is an immediate consequence of Theorem 1 and of inequality [7] $\omega \leqslant$ $1+\sqrt{2}$, so we shall prove Corollary 1 :

It is known [2] that for any convex set $K$,

$$
d \omega \leqslant 2 A
$$

and if $2 \omega \leqslant \sqrt{3} d$, then equality holds when $K$ is a triangle with basis $d$ and height $\omega$.

Corollary 1 now follows immediately from (3), since

$$
(\omega-\sqrt{2}) d^{2} \leqslant(\omega-\sqrt{2}) \frac{4 A^{2}}{\omega^{2}} \leqslant 2 \sqrt{2} A
$$

The equality occurs here when and only when $K=T_{\varepsilon} \in \mathcal{T}$, since it is easily seen that for all $T_{\varepsilon} \in \mathcal{T}$, the inequality $2 \omega\left(T_{\varepsilon}\right) \leqslant \sqrt{3} d\left(T_{\varepsilon}\right)$ holds.

\section{REFERENCES}

[1] W. Blaschke, Kreis und Kugel (Chelsea, New York, 1948).

[2] T. Bonnesen and W. Fenchel, Theorie der Konvexen Körper (Springer, Berlin, 1934).

[3] P.R. Scott, 'A lattice problem in the plane', Mathematika 20 (1973), 247-252.

[4] P.R. Scott, 'Area-Diameter relations for two-dimensional lattices', Math. Mag. 47 (1974), 218-221.

[5] P.R. Scott, 'A family of inequalities for convex sets', Bull. Austral. Math. Soc. 20 (1979), 237-245.

[6] P.R. Scott, 'Area, width and diameter of planar convex sets with lattice point constraints', Indian J. Pure Appl. Math. 14 (1983), 444-448.

[7] P.R. Scott, 'On planar convex sets containing one lattice point', Quart. J. Math. Oxford 36 (1985), 105-111.

[8] P.R. Scott and P.W. Awyong, 'Width-diameter relations for planar convex sets with lattice point constraints', Bull. Austral. Math. Soc. 53 (1996), 469-478. 
Departamento de Matemáticas

Universidad de Murcia

30100-Murcia

Spain

e-mail: mhcifre@fcu.um.es

salsegom@fcu.um.es 